\title{
Spectroscopic Studies of Binary and Multiple Star Systems
}

\author{
R. F. GRIFFIN \\ The Observatories, Madingley Road, Cambridge, CB3 OHA, UK
}

There can be few subjects that benefit more than binary stars do from complementary approaches. Consider their orbits, for an initial example. By 'visual' methods - by which I mean all methods of obtaining angular resolution on the sky, including the modern techniques of optical interferometry - one can determine an orbit, but with the scale known in terms of angular measure only. By spectroscopic (radial-velocity) methods one obtains the linear scale of the orbit, but with those techniques the inclination to the line of sight is indeterminate. In order to find the complete characteristics of the orbit in three dimensions - as is essential in order to find those most important data, the masses of the component stars - one needs to utilize both methods. It is, accordingly, clearly of great value to have a meeting like this that is objectoriented rather than technique-oriented; it is a long time since there was a meeting specifically devoted to binary stars, and especially in view of the great advances that have taken place in observational techniques of all sorts in recent years it is more than timely that we should have this meeting now. We are certainly very grateful to Dr. McAlister and his colleagues for arranging it and holding it at this beautiful venue.

The visual binaries that are most likely to be within the useful reach of spectroscopists are those of the shortest periods, implying at least statistically those of the smallest angular separations. In that connection we should particularly remember Finsen as having made as great a contribution as anyone, at least until the development of speckle interferometry and the efforts of people present here today, to the discovery and measurement of very close visual systems. I have a quotation to read to you from Finsen in the report (Lippincott 1962) of IAU Symposium 17, on Visual Double Stars, held in Berkeley in associaion with the IAU General Assembly there in 1961. Incidentally, that was even earlier than any of the conferences pictured in the next room; yet, among the 34 participants, there were four who are here with us today. They are Messrs. Couteau, Dommanget, Heintz, and Worley. We must congratulate them on their longevity and on maintaining their interest in our sub ject over such a long period of time. [Applause]

Before I read Finsen's remarks to you, I have a little story to tell against myself. Even longer ago than the Berkeley symposium, I had the good fortune one day to visit the Union Observatory (as it was then called) in Johannesburg. Although I was only a student at the time, Finsen received me very cordially and took me round, and indeed he and Mrs. Finsen were kind enough to entertain me to lunch. Of course he showed me the $26 \frac{1}{2}$-inch refractor and the eyepiece interferometer with which he made all those amazing discoveries and measurements of excessively close double stars. Not being interested at the time in double stars or knowing anything about Finsen's work on them, and supposing in my youth and naïveté that the only worthwhile techniques in modern astron- 
omy were spectroscopic, I viewed rather patronizingly such simple equipment, though no doubt I tried to look wise and say "How interesting" and suchlike polite remarks. In more recent times I have felt grieved that the privilege I was accorded that day was so completely wasted on me: I felt none of the awe with which, if only the experience could be repeated, I would - and indeed do now regard that simple equipment and that great observer, a combination which routinely allowed not only the measurement but even the discovery of binaries far closer than a telescope of that size had any business to be able to resolve.

Now at last I come to the quotation that I was going to read. It is not a direct quotation, since the report of the Berkeley Symposium is in the form not of a series of individual papers but of a summary written in the third person by an editor; but what Finsen is reported as saying is as follows. "When Michelson, Anderson, and Merrill made their first experimental interferometer observations of double stars more than forty years ago, they were hoping to resolve double stars, in Michelson's words, 'beyond the powers of the largest telescopes.' One would have expected, therefore, that their brilliant success in resolving the spectroscopic binary Capella with the 100-inch reflector would have been energetically followed up, with the promise of reducing and perhaps even closing the gap between spectroscopic and visual binaries." And Finsen then goes on to lament that that had not happened, and explains rather pathetically that he was obliged to make troublesome interferometer measurements with his comparatively small telescope merely because a large telescope, which would enable measurements of the same stars to be made directly with much less effort, was not available for the purpose.

Finsen's remark about closing the gap between spectroscopic and visual binaries is a very significant one, which brings us back to the point I made at the outset of this talk - that the two techniques are complementary and that both are needed to solve orbits completely. In the last twenty-five years there has been a lot of progress, at least potentially, in narrowing the gap from both sides. The development of speckle interferometry brought double stars having angular separations of only 30 or 40 milliseconds of arc within the range of routine measurements on large telescopes, and observing time on such telescopes was made available for such measurements although it had not normally been granted for work on 'visual' double stars previously. The speed, precision and scope of speckle measurements gave immediate promise of a flood of orbits for binary stars with much shorter periods than had been accessible before, and the further development of interferometry with unfilled apertures promises another great advance in the same direction. On the spectroscopic side, the introduction of direct cross-correlation procedures for measuring spectral Doppler shifts has provided a great improvement in the ease with which accurate radial velocities can be determined, as well as enabling good velocities to be achieved for objects a thousand times fainter than before. It has thereby become practicable to follow the velocity changes of numerous slow spectroscopic binaries whose amplitudes are at most a few kilometers per second, and all that is now required is persistence - a characteristic that has not been a particular strength of the radial-velocity fraternity in the past - to document many orbits with periods well into the range that used to be regarded as the province of visual doubles.

By way of showing what has actually been accomplished in terms of creating a useful overlap between the domains of visual binaries, on the one hand, and 


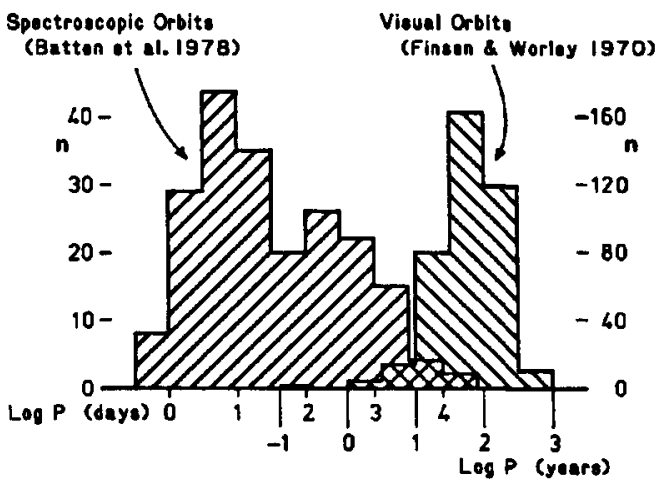

FIGURE 1. Period distributions of spectroscopic and visual binaries having tolerably well determined orbits, taken from the comprehensive catalogues cited. The Figure shows the situation before cross-correlation methods contributed to spectroscopic orbits or speckle interferometry contributed to 'visual' orbits. Notice how small is the overlap (cross-hatched) between the two distributions.

spectroscopic binaries, on the other, I have prepared a few viewgraphs showing the frequency of published orbits of different types against the logarithm of the period. The first one (Figure 1) illustrates the distribution of 204 orbits taken from the Seventh Catalogue of the Orbital Elements of Spectroscopic Binaries (Batten et al. 1978). I went back to the Seventh Catalogue rather than use the Eighth (Batten et al. 1989) because it fairly documents the situation at about the time that orbits derived by the cross-correlation method began to pour into the literature. In order to restrict the sample to tolerably well determined orbits, and to stars of the types that could be measured by cross-correlation (for comparison with subsequent samples), I have only plotted orbits of qualities $a$ to $c$, rejecting the worst two of the five recognized qualities, and have only taken systems with at least one component of type F5 or later. They are divided into period bins each half a logarithmic unit wide, and you see that they form a skew distribution with its maximum in the 3-10-day bin and a tail extending towards long periods. Here is a similar histogram [added to Figure 1 in this published version of the talk - ED.] plotted for the Finsen \& Worley (1970) Third Catalogue of Orbits of Visual Binary Stars; again I have restricted the sample to the three best of the five qualities into which the orbits were divided, but in this case I have not imposed any restriction as to spectral type. Again, I deliberately refrained from using the most recent orbit catalogue, in order to show the position before speckle measures contributed to the situation. The maximum frequency is in the 30-100-year bin. It is only too apparent that there is almost no overlap between the two distributions: only about $3 \%$ of the spectroscopic orbits fall within the range of periods having a significant population of visual binaries, and exactly the same thing is true in the reverse comparison.

So much for the historical position - now to see how it has changed as a result of the subsequent technological developments. Here (Figure 2) is the distribution of the periods of 'visual' orbits published in the last six issues of the Commission 26 Circulars so helpfully compiled and distributed by Dr. Couteau. 
After all this build-up you will be disappointed - as $I$ certainly was - to see that the most popular periods show a marked shift in the wrong direction, to still longer periods, still further removed from the domain of spectroscopic binaries! The explanation probably lies in the fact that Dr. Couteau does not risk grading the orbits in his Circulars but reports whatever is published. All those orbits with extraordinary periods, many of them far longer than the intervals that have elapsed since the respective objects could first have been measured, must be very shaky, and if they had appeared in a catalogue and been graded they would have been filtered out by the quality restriction that I imposed on the Catalogue sample.

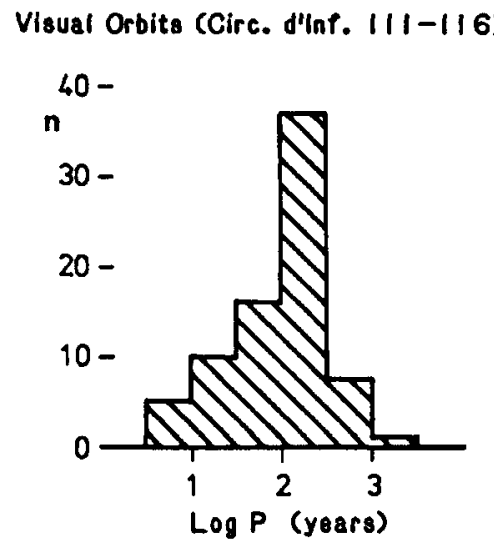

FIGURE 2. Period distribution of orbits reported in the six most recent Circulars issued by Dr. Couteau on behalf of IAU Commission 26.

To put the present position in the most optimistic light, I have tried a comparison of my own orbits with the Georgia State ones. Perhaps I ought to make an excuse for the apparent self-advertisement here, on the grounds that no claim is being made that my orbits are better than other people's: the fact is merely that I have the very dubious advantage of being so much older than other cross-correlation practitioners that my orbits extend to longer periods than those that other people have had time to reach! As a reasonable sample of my products I chose the orbits given in the first 100 papers published in the series in Observatory - an easy choice because I had already compiled their period distribution for a recent synopsis (Griffin 1991a), where indeed a histogram analogous to Figure 1 will also be found. The distribution of the 100 orbits (shown in Figure 3) is, as you see, very much shifted towards long periods in comparison with the Catalogue sample, with a maximum frequency in the 1000-3000-day bin and an appreciable population even in the next bin extending up to 10,000 days. It obviously represents a very significant start towards closing from the spectroscopic side the yawning gap seen between the two period distributions portrayed in Figure 1: the maximum frequency is shifted by $2 \frac{1}{2}$ logarithmic units out of the $3 \frac{1}{2}$ that separate the maxima in Figure 1 (actually the separation is a little over $3 \frac{1}{2}$ units, because a year is a little over $10^{2.5}$ days). To see how the 'visual' orbits have fared as a result of the routine application of the speckle method, I looked through the literature for the orbits published by Hal McAlister and his colleagues; their period distribution has been added to Figure 3. 


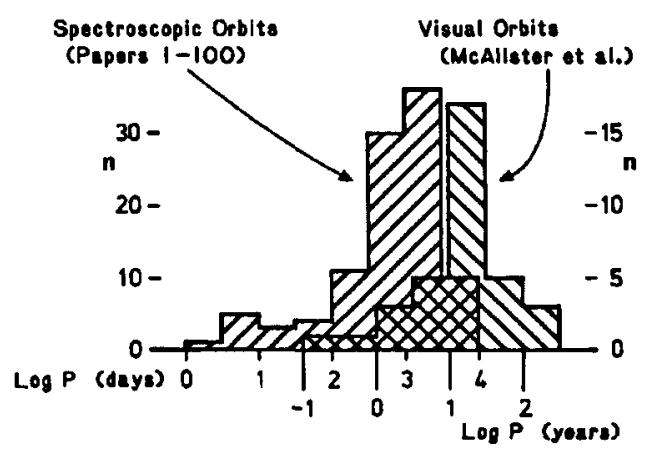

FIGURE 3. Period distributions of the author's spectroscopic orbits published in his series of papers in Observatory, and of the orbits derived by the Georgia State University speckle-interferometry group. The two distributions could be expected to overlap more than any other pair of samples of spectroscopic and 'visual' orbits; but despite the fact that the frequency maxima are much closer together than in Figure 1, the actual overlap between the distributions is still disappointingly small.

It must be confessed that I find this comparison very disappointing: I quite expected to find now a substantial overlap between the two histograms. Certainly there is appreciably more overlap in Figure 3 than in Figure 1, but the bins containing the principal contributions to the two distributions are still completely separated. What can be done about it? Obviously we must try harder! I have to admit that I personally can't do very much more, because life simply isn't long enough. To populate the next bin beyond where my present orbits reach will take too long. Even if I knew which stars would make profitable objects to study, with logarithmic periods (days) in the range 4 to 4.5 , and I started observing them now, I would be 140 years of age before they had come round a single cycle - and I'm afraid I shall have other things to do before then! The conclusion has to be that, if the spectroscopists and 'visual' observers are to talk about many of the same stars at all soon - while most of us at this Colloquium are still alive - the main fresh advance will have to come from the 'visual' side. Actually, there are very encouraging signs that that is exactly what is on the point of happening: already resolutions of a few milliseconds of arc are being obtained, and I believe we are going to hear of plans to trespass into the microsecond régime. If baselines of hundreds of meters can really be achieved, then even binaries in the traditional range of spectroscopic periods will become resolvable, with dramatic implications for the advance of our subject.

Although I have probably projected a rather defeatist image concerning the possibility of determining spectroscopic orbits with periods above 10,000 days, I ought perhaps to mention that a few such orbits are within realistic prospect already; after all, it is more than 9,000 days since I began the photoelectric work, although it is a matter for regret that I did not instantly start work on all the long-period objects that I know about now! A good example is this one of $\kappa$ Persei (Figure 4), whose period of 10,400 days $\left(28 \frac{1}{2}\right.$ years) is determined within a standard error of only two months. My own observations now cover about three-quarters of a cycle, and the early Lick measurements of 


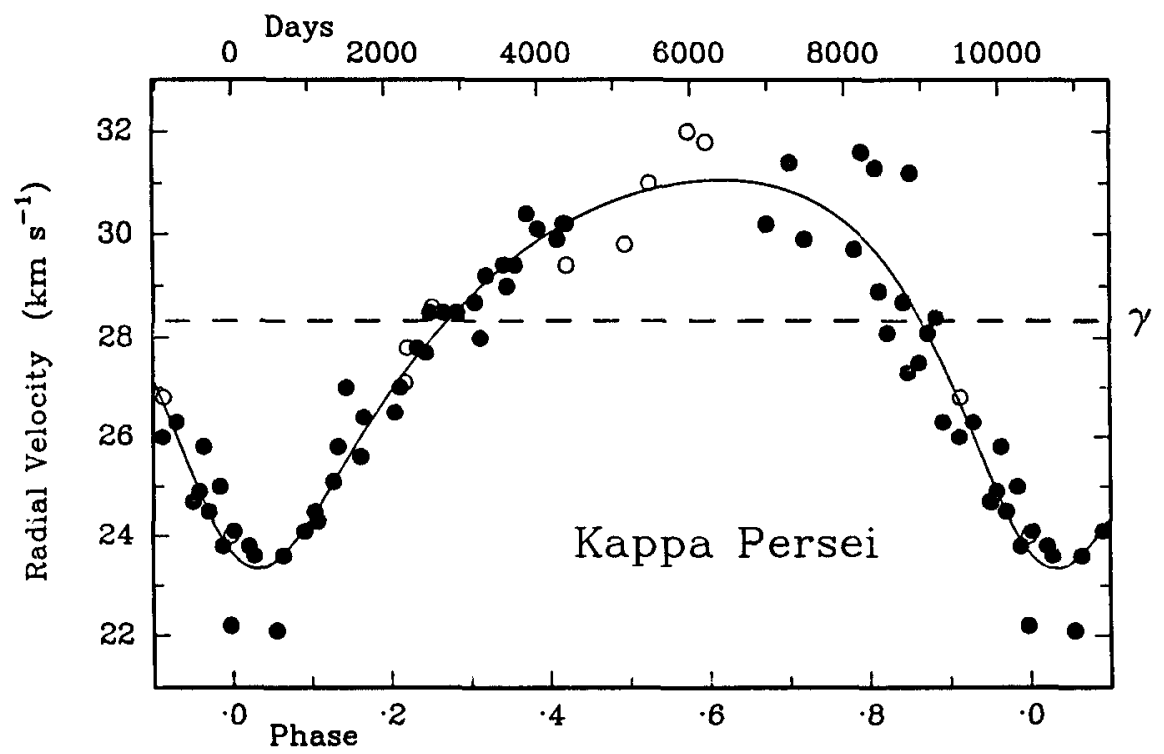

FIGURE 4. Preliminary orbital radial-velocity curve for $\kappa$ Persei. The solid dots are the author's measurements made since 1969; the open circles are Lick measurement. taken in 1899-1922. The period is about 10,400 days ( $281 / 2$ years).

this fourth-magnitude star form a nice complement two cycles ago. Another one, which depends upon my own observations alone, is HR 4593 (Figure 5). The period appears to be about 12,000 days or 30 -odd years, but it is not very well determined at present; of course the observations do not by any means cover the whole orbit, and the only reason that one can derive a sensible orbital solution already is that we happen recently to have passed a periastron passage and have witnessed both nodes. Unfortunately the early observations were rather few and ragged, and the orbit cannot really be well determined until after the next periastron passage, which will involve a wait of about thirty years. In addition to stars such as those for which I have just shown orbits, there are of course others that have undergone slow changes in radial velocity but which do not appear to be anywhere near to completing an orbital cycle yet: all that can be said is that a start has been made on them observationally, and if they are observed sufficiently persistently then some at least of them will in due course furnish orbits of longer periods than any yet determined spectroscopically.

I want now to turn to a somewhat different topic and describe some of the work that my wife and I - mainly my wife! - have been doing on compositespectrum binaries, which have provided a wonderful opportunity for the exercise of complementary techniques. Such binaries, according to the restricted definition that we adopt for them, consist of a luminous late-type star (a $G$ to $M$ giant or supergiant) with an A- or B-type companion that is on or near the main sequence. The ones that we can profitably investigate are those in which the luminosities of the two components are not grossly dissimilar in the traditional optical region, particularly the violet and near-ultraviolet where we can 


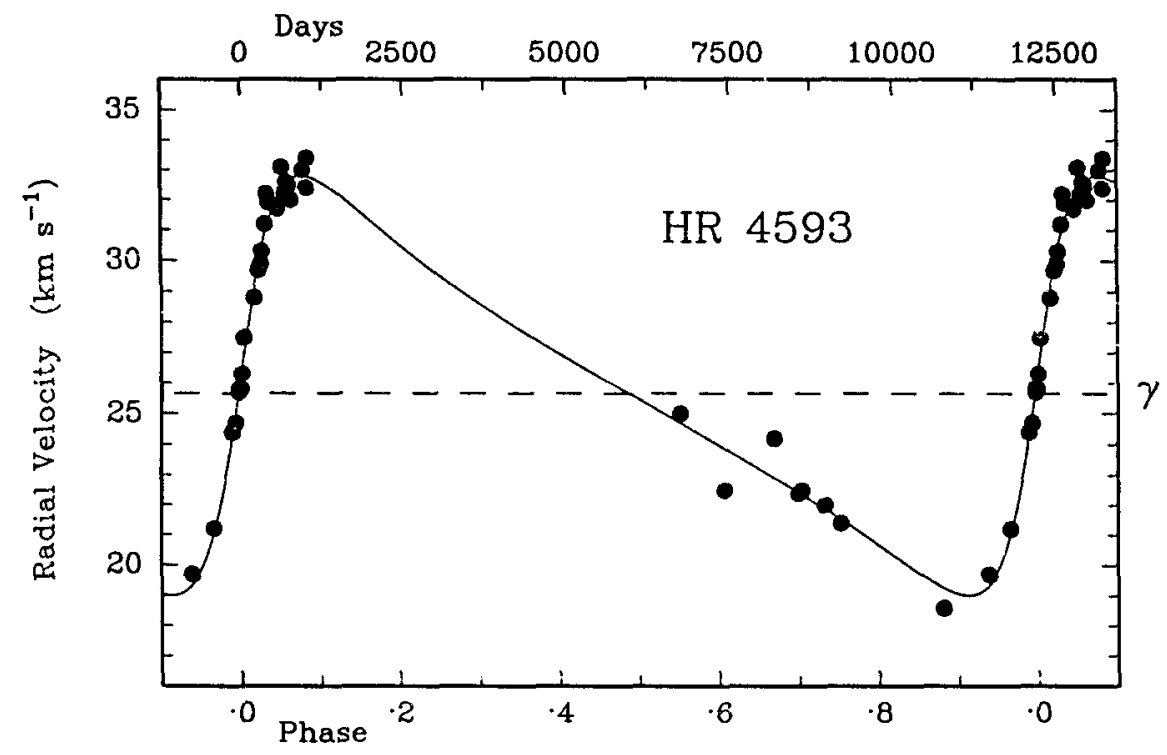

FIGURE 5. Preliminary orbital radial-velocity curve for HR 4593, from the author's observations taken since 1973 . The period appears to be about 12,000 days (33 years).

record the spectra photographically. Some of this work is already in print, and pictures of spectra similar to some that I am going to show will be found in our series of papers on composite spectra in the Indian journal (e.g., Griffin 1986) and on $\zeta$ Aurigae stars in the A\&A (e.g., Griffin et al. 1990).

Composite-spectrum binaries have been greatly neglected in the past, perhaps largely because their very compositeness rendered them an almost hopeless proposition for any sort of quantitative spectroscopic study. There are many bright examples whose orbits, even, are unknown, and others whose published orbits are erroneous. The first thing to do is to determine reliable orbits, and I content myself with showing two of them here. This one (Figure 6) is of McAlister 2, which has a period of 2090 days or 5.72 years, with a standard error of only two days. There are three orbits for it in the literature already, all of them wrong: a spectroscopic one of 226 days, and two derived from speckle interferometry with periods of 11-point-something and 12-point-something years to save embarrassment I won't mention any names! McA 2 has shown considerable variations of position angle and so cannot be an eclipsing system. On the other hand this one, McA 12 (Figure 7), which has spectroscopic orbits of 220 days and 212 days in the literature, has a well-determined period here of 6135 \pm 11 days - about 17 years. All those star symbols along the top of the plot show the phases of Lick spectrograms that were taken on purpose to determine the orbit for this system but have never been measured. It seems a great pity that relevant material on this and other stars should lie dormant in such a way through lack of measurement. There were several speckle measures of McA 12 before the periastron passage of 1984, and they showed no significant change in position angle, so it could well be that this object will prove to be an eclipsing 


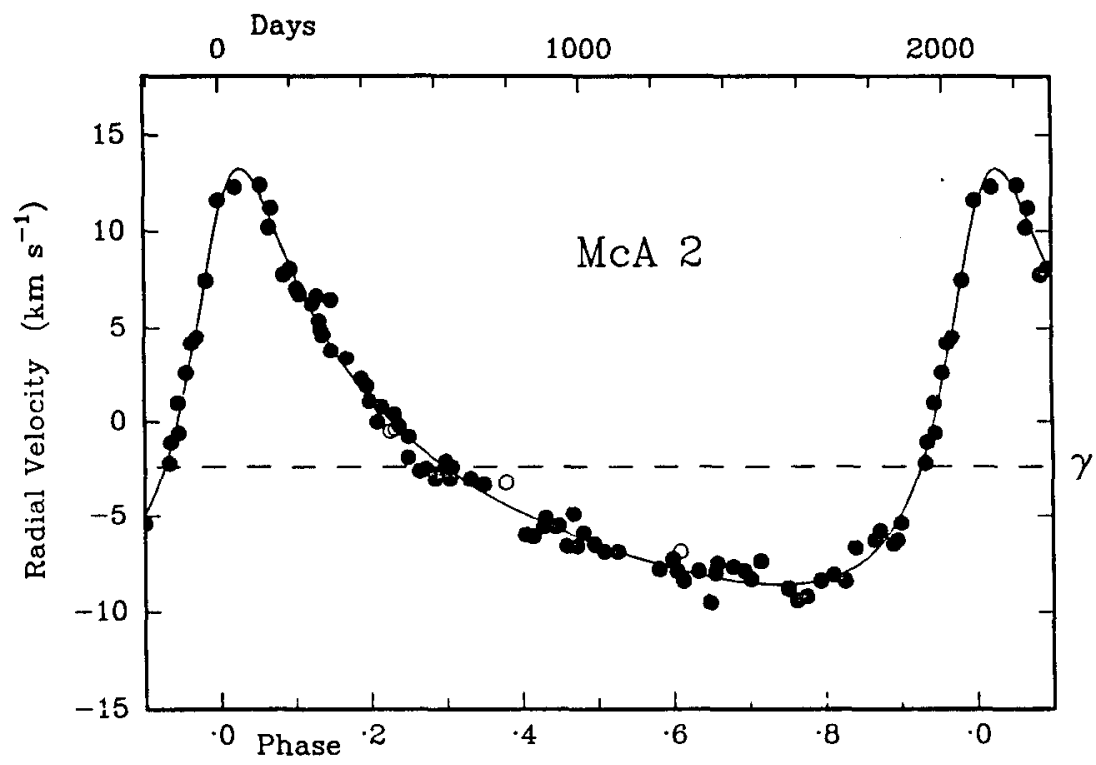

FIGURE 6. Radial-velocity curve for McA 2; the solid dots represent the author's observations, the open circles the early Lick measures.

system. Towards periastron it became too close for resolution, and it does not seem to have been measured since. Most unfortunately, I only derived the orbit just after the conjunction that took place in late 1986, and we have to wait till 2003 to see the next one; but long before then, no doubt, renewed speckle measures will have determined whether or not an eclipse is to be expected.

The technique we have used to complement the radial velocities (and sometimes the speckle measures) of these stars is spectroscopic. My wife has developed procedures, applied to spectra carefully digitized from photographic material, to subtract from the composite spectrum a spectrum analogous to that of the giant component of the system, and thereby to uncover the spectrum of the hot star. In typical composite spectra one ordinarily sees the hot component only as a sort of veiling of the lines of the cool star and a characteristic infilling of the late-type $\mathrm{K}$ line, but after subtraction one has a perfectly good spectrum that can be classified, measured for radial velocity, and used for all the purposes that a spectrum can normally serve. I may say that, although on these tracings showing a convincing example of the subtraction process it all looks very simple and straightforward (and in principle it is) it actually proved to be an altogether more difficult exercise than at first we expected to get results as good as these.

Quite by chance the first system that we wrote up, HR 6902 (Griffin 1986) proved to be an eclipsing binary. Once our suspicions on that score were aroused, we predicted the date of conjunction from the radial-velocity orbit, obtained observing time on the critical nights through the kindness of the DAO, and my wife (assisted by our son Rupert) went and observed the eclipse. As in the case of $\zeta$ Aurigae and other such systems where the eclipsing star is large and cool, chromospheric lines were seen just outside the duration of the bodily eclipse. By 


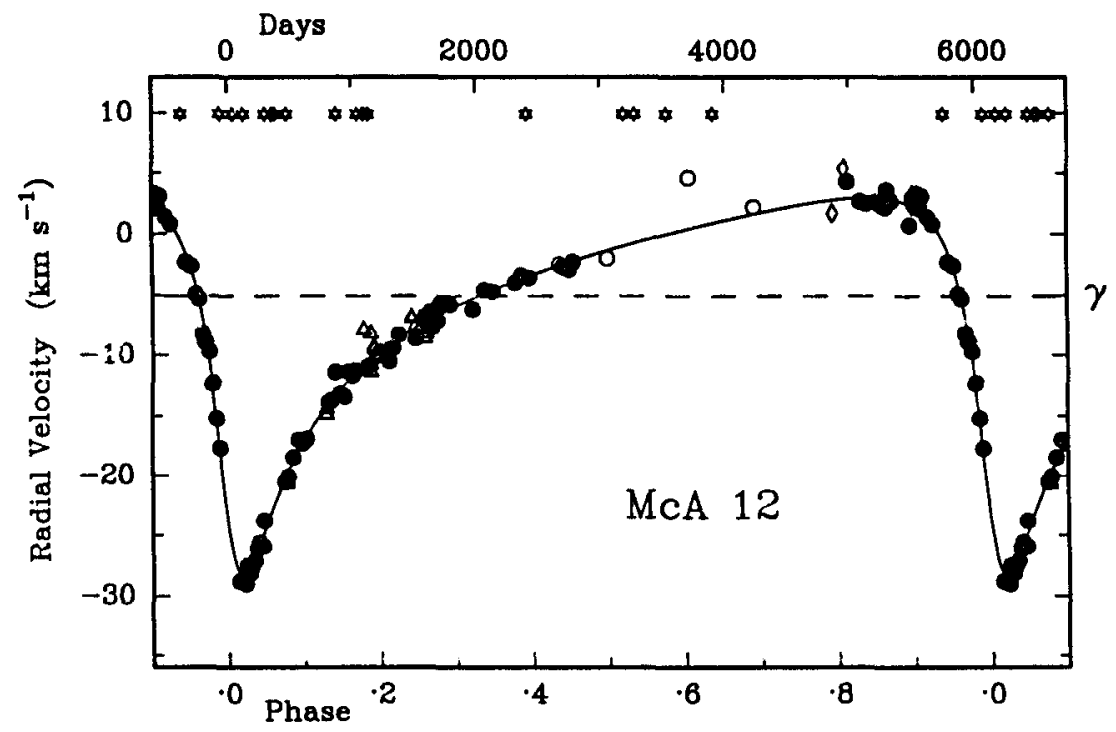

FIGURE 7. Radial-velocity curve for McA 12. Again, the solid dots are the author's own results, beginning in 1981 at phase .8; open symbols represent radial velocities from various sources in the literature. The star symbols near the top of the Figure show the computed phases of Lick spectrograms that were taken with a view to determining this orbit (and would still materially assist it) but have never been measured.

applying the subtraction technique to spectra taken during the chromospheric phase of the eclipse, it was possible to isolate the chromospheric spectrum an achievement that promised such benefits that we have made special efforts to observe other $\zeta$ Aurigae stars at eclipse times. You can see from the tracings that in the raw spectra the chromospheric lines, if visible at all, appear merely as enhancements in the cores of already-existing strong photospheric lines in a spectrum of daunting complexity; but in the subtracted spectra they appear as individually isolated lines set against the nice level continuum of the B-type spectrum. It hardly takes an expert to see what an advantage it is, in any investigation of the chromosphere, to start from the subtracted spectrum rather than the raw comnposite.

The $\zeta$ Aurigae binary that we are working on now is a particularly difficult one, 22 Vulpeculae. The special difficulty lies in the relative faintness of the late-B companion in comparison with the G-supergiant primary star. Clearly, in such a case there is very little 'signal' left when one has subtracted away the primary; and since in a subtraction it is the signal that is subtracted but the noise is nevertheless additive, one needs to start with material of high quality and high signal/noise ratio. Such material was obtained at the 2.2-meter coudé on Calar Alto. These spectra (which will be published in a paper shortly to be submitted to Astronomy $\mathcal{B}$ Astrophysics) show the situation on successive nights at ingress, and at a casual inspection they hardly look any different from one another! - which is not surprising, because it was not until attention was 


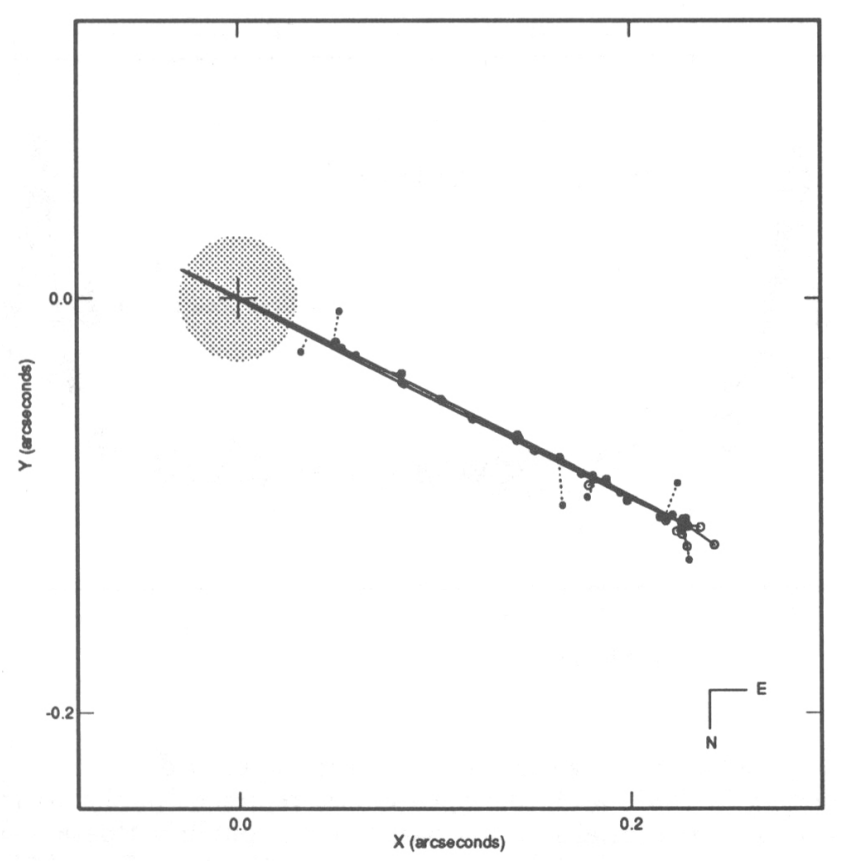

FIGURE 8. Apparent orbit of $\gamma$ Persei, derived from speckle interferometry. The $\gamma$ Persei system is a composite-spectrum binary consisting of a late-G giant primary with an A-type main-sequence companion in an orbit with a period of nearly 15 years. (Figure kindly provided by Dr. H.A. McAlister; see, for example, McAlister's poster on HR 1071 for a description of symbols used.)

drawn to $22 \mathrm{Vul}$ by the discovery of its true nature with IUE that its spectrum was noticed as being composite at all. Nevertheless, the same spectra after the total-eclipse spectrum (the G supergiant) has been subtracted, although noisy in comparison with the raw spectra, show very clearly the rapidly increasing richness of the chromospheric spectrum and the behavior of the individual lines as we look through deeper and deeper layers of the chromosphere on successive nights. They certainly constitute a very firm foundation on which to base an understanding of the chromosphere of $22 \mathrm{Vul}$.

The last of the composite-spectrum binaries about which I want to tell you today is $\gamma$ Persei: less than two years ago it was discovered to be an eclipsing system whose eclipses are detectable with the naked eye but had never been noticed before. An orbital period of nearly 15 years was determined by McLaughlin many years ago, and during the last cycle the speckle-interferometry people here have found that the orbit is presented almost exactly edgewise to the line of sight, making an eclipse a distinct possibility (Figure 8). Unfortunately neither the speckle measurements nor the radial velocities predicted the time of conjunction at all accurately. However, the star had been on the Cambridge radial-velocity program for about ten years, and it was clear (Figure 9) that, once the velocity 


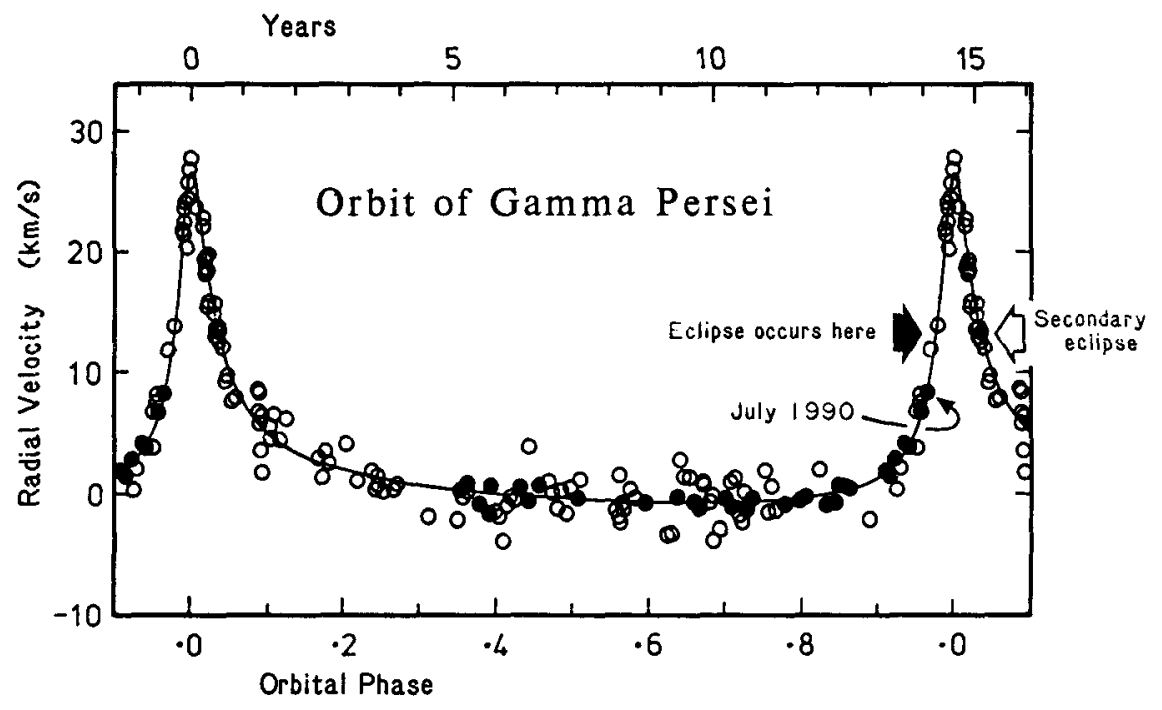

FIGURE 9. Radial-velocity curve of $\gamma$ Persei; the solid dots are the author's observations, starting in 1981 , while the open circles represent published measurements from earlier cycles. The orbital solution based on the published data was not by any means precise enough to allow the date of conjunction (the date of possible eclipse) to be predicted with the reliability necessary for the setting up of an observing program; the Figure shows how, owing to the nature of the orbit, it was not until July 1990 that the new observations could determine the phase well enough to give a good prediction. By that time the eclipse was too imminent for observing time to be sought on any suitable telescope through regular channels.

had fairly started up the rise towards maximum, it would be possible to predict the date of conjunction reasonably well. I was also pretty well convinced by the speckle observations that an eclipse would take place. As related in an article in Sky \& Telescope last year (Griffin 1991b), an exciting observing campaign ensued, in the course of which I obtained spectra of $\gamma$ Per on 29 out of 30 consecutive nights, first at Calar Alto and then - after a rapid transit in which I did not miss a single night - at Palomar, where I was very lucky to manage to beg morning twilights at short notice for weeks on end at the 200-inch telescope. In the event there was an eclipse, which was total for a week and had partial phases each lasting rather more than a day. Not only did I obtain a complete spectroscopic record of it, with spectra at $2 \AA / \mathrm{mm}$ every night, but I had alerted photometrists to the likelihood of the eclipse and they too obtained a good record of it, as you see from this sketch of the light-curve (Figure 10). The story of $\gamma$ Persei seems to me to be a particularly good example of what can be achieved by the convergence on a single problem of a whole panoply of complementary techniques, including in this instance speckle interferometry, radial velocities, photographic spectroscopy and photoelectric photmetry. 


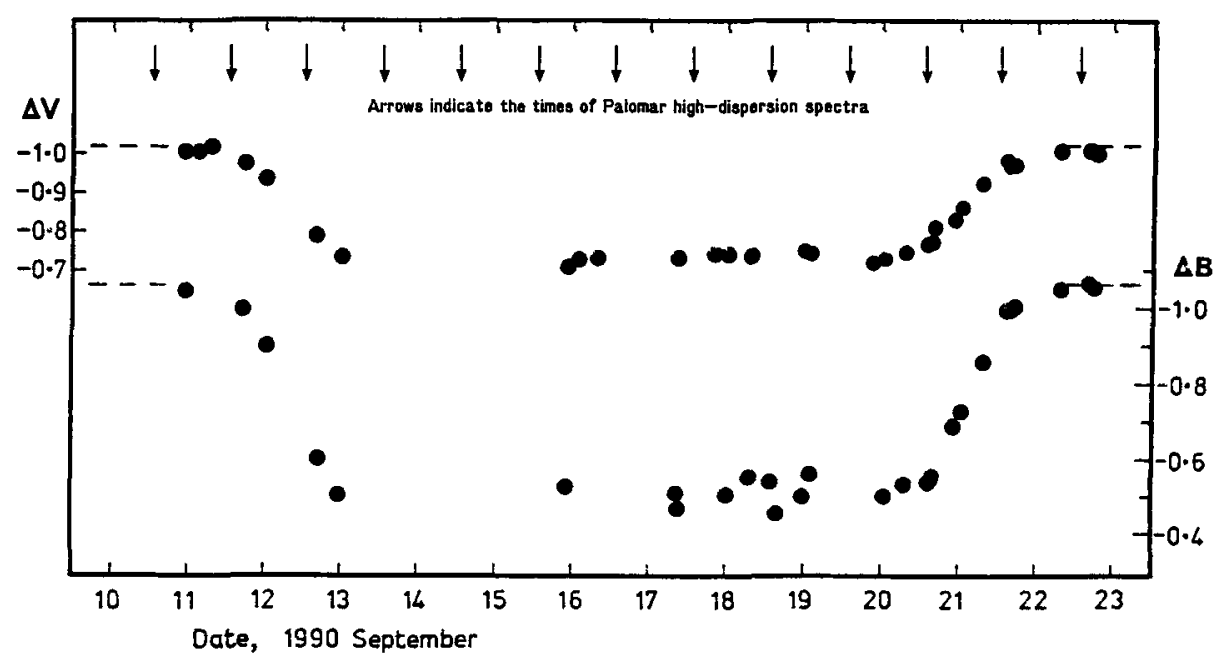

FIGURE 10. Photometric record of the eclipse of $\gamma$ Persei in 1990, the occasion of its discovery which was predicted on the basis of the data shown in Figures 8 and 9. Photometry was provided by nine observers (Messrs. Arai, Houchen, Krisciunas, Ohshima, Pray, Schröder, Snyder, Tokoro, and Williams) who had been alerted by the author to the likelihood of the eclipse. Meanwhile the author himself obtained daily high-dispersion spectrograms throughout the eclipse; he used the coude spectrograph of the 200-inch telescope in morning twilights, which had very kindly been put at his disposal at short notice by Palomar Observatory and the regularly scheduled observers.

\section{REFERENCES}

Batten, A.H., Fletcher, J.M., \& Mann, P.J. 1978, Publ. DAO, 15, 121

Batten, A.H., Fletcher, J.M., \& MacCarthy, D.G. 1989, Publ. DAO, 17

Finsen, W.S. \& Worley, C.E. 1970, Republic Obs. [Johannesburg] Circ., 7, 203

Griffin, R.F. 1991a, Observatory, 111, 291

Griffin, R.F. 1991 b, $S \mathcal{S T}, 81,598$

Griffin, R. \& R. 1986, J. Astrophys. Astr., 7, 195

Griffin, R.E.M., Griffin, R.F., Schrōder, K.-P., \& Reimers, D. 1990, A\&A, 234, 284

Lippincott, S.L. 1962, PASP, 74, 5

\section{DISCUSSION}

EVANS: I'm impressed with the subtracted spectra you showed (having tried it myself with nothing like such clean results). What kind of plates did you use?

GRIFFIN: They were trailed IIa-O plates.

McALISTER: I would just like to remark that interferometers, operating over baselines of hundreds of meters, will soon (in this decade!) eliminate the gap between the classical and visual binaries, which you have so nicely illustrated. Speckle has been a kind of "cantilever" into this gap, but long-baseline interferometers will resolve the great majority of the known spectroscopic binaries. 